\title{
ONE MASK ONE EXPOSURE IN-SITU MAGNETIC ACTUATOR FABRICATION METHOD
}

\author{
S. E. Chung, J. Kim, S. Min, L. N. Kim, and S. Kwon \\ Seoul National University, Seoul, South Korea
}

\begin{abstract}
We demonstrate in-situ magnetic actuator fabrication technique via one-mask one-exposure method. A single magnetic microstructure or magnetic actuator is photopatterned from magnetic nanoparticles (MN) containing ultraviolet (UV) light curable resin in PDMS microfluidic channels. Oxygen inhibition layer generated from the PDMS surface allows magnetic microstructure to move and float without a sacrificial layer concurrently with the fabrication process. Thus, we only need one-time UV patterning with one-mask to fabricate a single complex magnetic actuator. We generated various magnetic structures such as both anchored and floating actuators with the above simple one-mask one-exposure system. These magnetic structures are actuated by external permanent magnet controlled outside of the channel. Overall magnetic actuator fabrication and control are take place in the same area without extra step to transfer the position from the fabrication site to the actuation site. Therefore, the entire process is very simple and fast enabling in-situ fabrication and real-time actuation.
\end{abstract}

\section{INTRODUCTION}

Electroplating and micromachining is a fundamental process to manufacture general magnetic actuators in conventional microelectromechanical systems (MEMS) technology. Electrodeposition of magnetic materials and micromachining such as etching after lithographical patterning form metal layers for various magnetic actuators [1-2] and these are very popular methods due to higher microscale accuracy. However, this conventional MEMS technique to fabricate actuators is very complex and time-consuming method. It is necessary to deposit sacrificial layer and remove it after the patterning [3] to fabricate a simple movable magnetic actuator, such as electrostatic clamping structure [4], or anchored cantilever beams [5-6].

Instead of using top-down approach by micromachining the bulky metal solids, fabrication of magnetic actuators from magnetic nanoparticles would be easier, simpler and more flexible. Recently, magnetic nanoparticles are encapsulated in a polymer matrix by photopolymerizing magnetic beads within UV curable resin [7-8]. By embedding these magnetic nanooparticles in hydrogels via photopolymerization, various elastic magnetic actuators are fabricated due to its flexibility of solidified polymers.

In this paper, we have developed very simple and fast fabrication method of magnetic actuators via one-mask one-exposure lithography. With optofluidic maskless lithography (OFML) technique [9], a magnetic microactuator, or a magnetic microstructure, is directly photopatterned from the mixture of magnetic nanoparticles (MN) and ultraviolet (UV) light curable resin within a second. The PDMS channel used as fabrication and actuation sites provides good environment for magnetic microstructures to move and float, because oxygen inhibition layer is generated during the photopolymerization process [10]. The inhibition layer allows magnetic actuators to be fabricated without sacrificial layer. Different from magnetic actuator fabrication technology in MEMS field, our technique only execute one-step lithography to fabricate various actuators even a three-dimensional anchored cantilever beam using PDMS patterned glass substrate by taking advantage of inhibition layer. Therefore, we only need one-time UV patterning with one-mask to fabricate a single complex magnetic actuator.

Both floating actuators in microfluidic channels and anchored actuator outside of the channel such as magnetic microcantilever are easily fabricated using our simple one-mask one-exposure in-situ fabrication technique. Each photopatterned microstructure contains MN embedded inside, and those nanoparticles react to the external magnetic force and cause the polymeric microstructure to actuate. These magnetic structures are easily actuated by controlling external permanent magnet outside around the channel. The actuators move through the magnetic field directions formed by the applied magnet. Free-floating actuators, bounded actuators using anchors of the microfluidic channel and railed actuators in railed microfluidic channels [11] are presented as actuation demonstration of in-situ fabricated magnetic structures. Furthermore, selective magnetic actuation is also demonstrated from heterogeneous assembly of magnetic structures and non-magnetic structures.

\section{EXPERIMENTALS}

Fabrication and control of magnetic microactuators

A magnetic actuator, which is $\mathrm{MN}$, magnetite $\left(\mathrm{Fe}_{3} \mathrm{O}_{4}\right)$ embedded microstructure, is fabricated by optofluidic maskless lithography (OFML) within microfluidic channels. As shown in Fig. 1 , two types of magnetic actuators are fabricated; floating actuators (Fig.1(a)) and anchored actuators (Fig.1(b)). First, MN are mixed with photocurable resin and introduced into the prepared microfluidic device. Then, we polymerize the liquid-phase $\mathrm{MN}$ resin using one-mask one-exposure process via OFML system. The thickness of the actuators is determined by the height of the channel and the lubricating inhibition layer[10]. During the photopolymerization, $\mathrm{MN}$ are embedded in the polymeric microstructures, thus it enables that the magnet pulls the fabricated microstructures due to embedded MN. Note that solidified microstructures containing $\mathrm{MN}$ are in-situ fabricated magnetic actuators. (a)

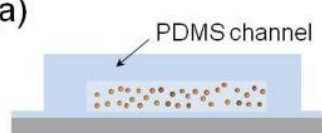

Introduce $M N$ containing UV curable resin

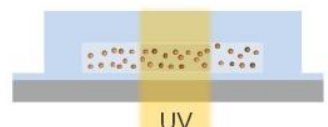

UV

One mask/one exposure

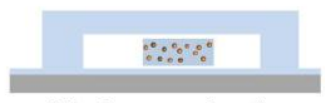

Wash uncured resin with buffer solution (b)

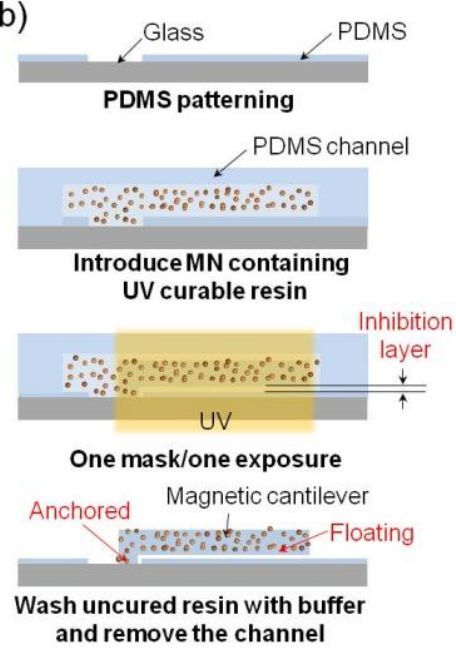

Figure 1. In-situ fabrication process of magnetic nanoparticles $(M N)$ embedded microstructures, which are magnetic microactuators, using one-mask and one-exposure process 
General fabrication process of floating actuators and anchored actuators is similar except the substrate types. The substrate of floating actuators is PDMS coated slide glass to generate free-floating photopolymerized structures due to oxygen inhibition layers [10]. Oxygen inhibition layer enables that cured polymeric structure on the PDMS layer does not adhere to the substrate allowing free actuation using external permanent magnet. However, the substrate of the anchored actuator is patterned PDMS coated slide glass, meaning that some portion of the substrate is not coated with PDMS. Without PDMS layer, synthesized part sticks to the glass, functioning as an anchor.

After the fabrication, we finally wash uncured MN containing UV curable resin with buffer solution, usually ethyl alcohol. For the anchored magnetic cantilever beam, we remove the channel for further actuation process.

Conventional cantilever beam fabrication technology requires complex fabrication process to make anchors and beams because sacrificial layer is necessary to get bendable cantilever beam. However, our magnetic actuator fabrication technique enables one-step fabrication of anchored cantilever beam via one-mask one-exposure process without a sacrificial layer by taking advantage of oxygen inhibition layer in PDMS device. During the fabrication process, an anchor of the cantilever is adhered to a glass and a beam is generated on the PDMS region enabling free-deflection.

Figure 2(a) shows the anchored magnetic cantilever fabricated on the patterned PDMS glass substrate and its actuation by applying magnetic field. Due to the magnetic force applied perpendicular to the substrate, the cantilever beam bends toward the field as the end of the beam is anchored to the substrate.

The actual experimental results are demonstrated in Fig. 2(b) as sequential images. At the initial state, the cantilever beam free-floats on the PDMS coated substrate due to lubricating inhibition layer and the end of the beam is anchored at the glass substrate (Fig. 2(b) (Top)). When the low vertical magnetic field is applied, the cantilever is lifted up overcoming the mechanical torque to straighten the beam. In this process, only unanchored part of cantilever beam, which is floating part on the PDMS layer, moves up. Under the high magnetic field, it is lifted up to almost $90^{\circ}$ degrees as shown in Fig.2(b)(Bottom).

(a)

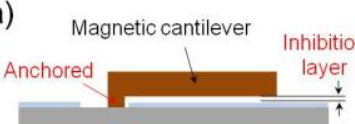

Fabrication of magnetic cantilever

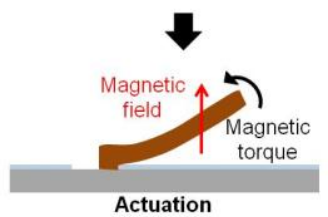

(b)

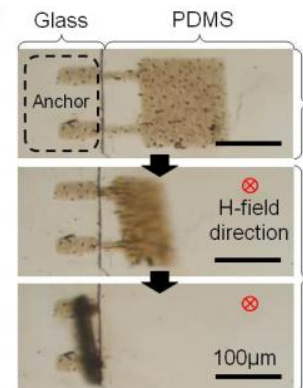

Initia state

H-field applied

Figure 2. Actuation of anchored magnetic cantilever (a) Schematic diagram of a magnetic cantilever actuation. (b) Magnetic cantilever actuation.

Figure 3 shows the experimental setup of in-situ floating magnetic actuators fabrication and actuation. The setup is composed of photopatterning part utilized by OFML system and magnetic controlling part using permanent magnet. OFML in-situ synthesizes magnetic microactuators by a patterned UV light projected from the DMD as shown in Fig.3(a). Each photopatterned microstructure contains $\mathrm{MN}$ embedded inside, and those nanoparticles react to the external magnetic force and cause the polymeric microstructure to actuate. Fabricated MN embedded microstructures are actuated by controlling external permanent magnet from the outside of the channel. The magnet induces a magnetic field to control actuators. The fabricated structures move along the magnetic field directions generated by the magnet.

Three types of floating magnetic actuators are demonstrated in the experiments (Fig.3(a)-(b)). Free-floating structure actuation uses free-floating MN embedded microstructures. Fig.3(c) shows Scanning Electron Microscope (SEM) images of free-floating magnetic structure. 100 120nm sized magnetic nanoparticles are fixed in a polymeric structure during photopatterning process Bounded actuator is fabricated at the PDMS anchor enabling bounded actuation such as rotation. Since one of basic movements is rotation, anchored actuators are applied to mixer or particle sorter at branches using rotational movement. Similarly, rail-guided actuator is fabricated on the rail and guided along the rail. Since railed microfluidics is an agile method to guide and assemble microstructures inside fluidic channels, it enables efficient magnetic structure sorting at the rail branches or various heterogeneous assemblies for selective actuation.

Free-floating magnetic actuators are affected from both applied magnetic field and flow stream through the fluidic channel. The magnetic field outside from the channel controls the transition direction of the free-floating actuators and initiates their movement. Then, the flow stream accelerates the transition of the actuators.

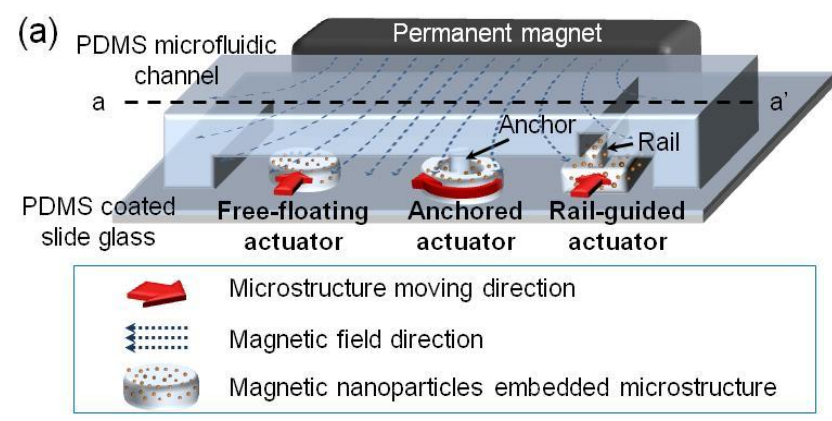

(b)

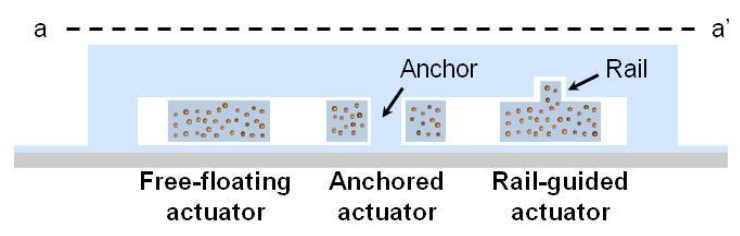

(c)

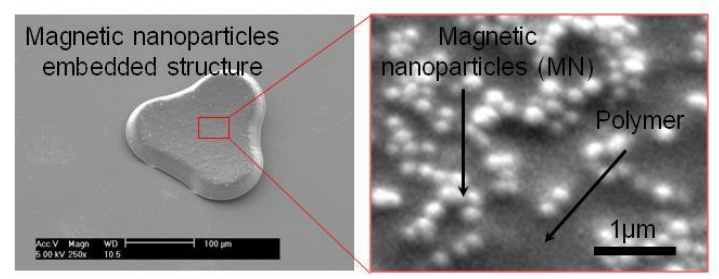

Figure 3. Experimental setup and fabricated magnetic actuators

\section{RESULTS AND DISCUSSION}

Figure 4 shows the free-floating actuation of $\mathrm{MN}$ embedded microstructure. As an initiate state, the actuator keeps its position within the channel as in Fig.4(a)(i). When the magnetic field is applied to the structure from the outside of the channel, the actuator transits to the field direction (Fig.4(a)(ii) and (iii)). In addition to the transition, the fluidic force is also applied from the inlet of the channel to complete the blocking of the branch. After the blocking, free-flowing polymeric microparticle is fabricated to demonstrate 
selective flowing due to blocked channel branch using free-floating magnetic actuator. The microparticle freely moves to the unblocked branch with the flow stream as shown in Fig. 4(b) or (c).
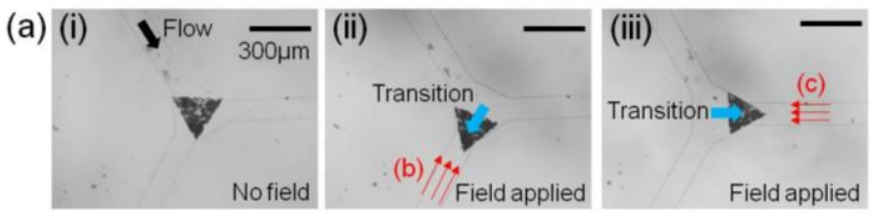

(b)
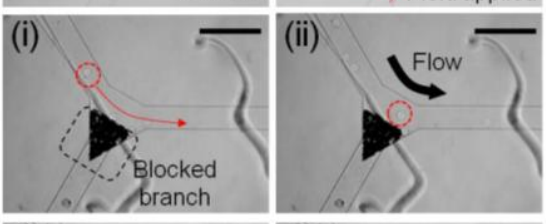

(c) (i)

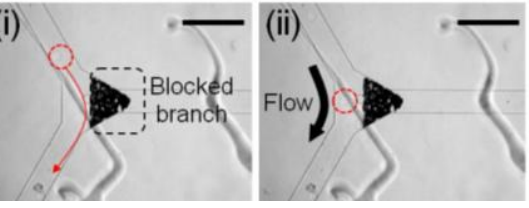

Figure 4. Free-floating actuation.

Bounded actuators are fabricated centering on the PDMS anchor, thus holes at the center of the structures are generated due to the oxygen inhibition layer of the PDMS anchor. These structures rotate in response to the induced magnetic field which rotates externally using permanent magnet around the channel.

Rotating mechanism enables various micromotor-type applications as demonstrated in Fig. 5. Figure 5(a)-(b) show free-floating particle sorter and transporter. Basically, the magnetic structure rotates bounded at the PDMS anchor while external magnetic field is rotating. If the rotating direction changes to the opposite way, the structure would also rotate in the opposite direction. This fact is applied to a magnetic sorter. In a two-phase microfluidic channel with a center PDMS anchor at the branch, the magnetic sorter receives a free-floating particle and sends it to upper branch of the channel by rotating in counterclockwise direction as shown in Fig. 5(a).

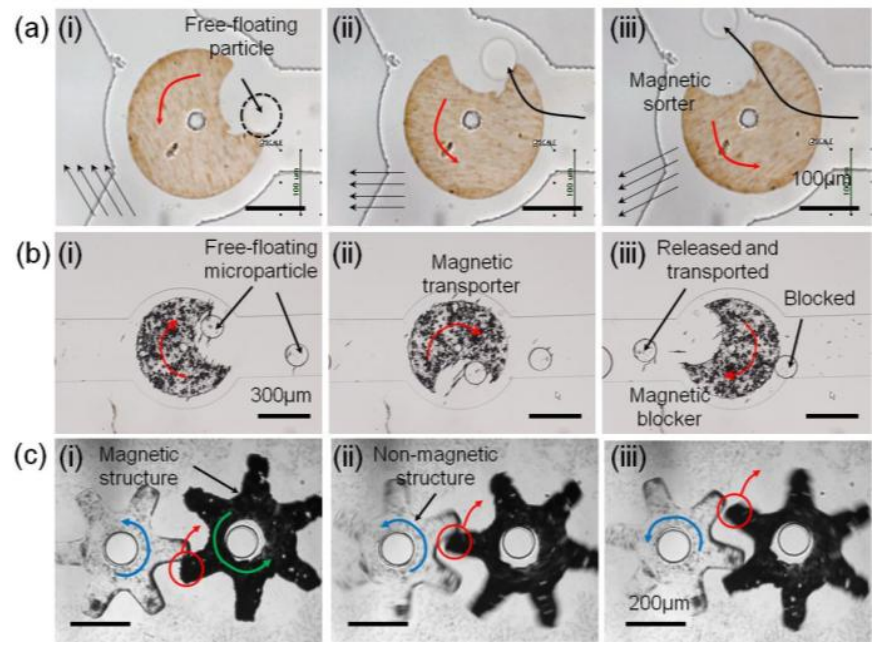

Figure 5. Full-bounded actuations of in-situ fabricated magnetic structures. The magnetic structures are fabricated centering at at PDMS anchors and rotate in response to the rotating magnetic field.

Free-floating particle transporter works in a similar way with the magnetic sorter. In one-way fluidic path, the magnetic transporter selectively releases free-floating microparticle and blocks others. Therefore, the actuator works as both transporter and blocker.

In addition to single magnetic micromotor actuation, heterogeneous actuation is also demonstrated in Fig. 5(c). Two toothed wheels, composed of magnetic structure and non-magnetic structure, go in gear with each other due to rotating magnetic field. In the system, magnetic wheel only responses to the applied magnetic field, thus non-magnetic wheel rotates engaging with the rotating magnetic wheel.

Figure 6(a) shows a basic magnetic control of MP embedded microstructures on the rails. Since the finned microstructures only go along the rail, moving direction of magnetic structures is controlled using magnetic field and the path of movement exactly same with the rails. By taking advantage of railed microfluidics, many interesting applications are proposed.

A sliding valve in two-phase microfluidics alternatively blocks a selective fluid as shown in Fig. 6(b)-(c). The position of magnetic structure on the rail depends on the field direction of external magnet. By effectively controlling the direction of external magnet, we selectively block unwanted flow streams using sliding valve moving along the rail and change the mainstream of the channel.

In addition to valve-type application, we effectively sort out lots of microstructures to designated branches by utilizing rails. Figure 6(d)-(f) show sorting mechanisms of the magnetic microstructures on the rails. At a branch point, the moving direction of each approaching magnetic structure is controlled by the direction of magnetic field. In Fig. 6(d), all microstructures move to the left branch due to the magnetic field applied from the bottom side of the channel, and in Fig. 6(e), all of them move to the right branch due to the field from left bottom side of the channel. However, magnetic field control also enables that each magnetic microstructure move to all different directions as shown in Fig. 6(f). Railed microfluidic based particle sorter system is a simple way of selecting the targeted rail for magnetic microstructures to ride in the second place. By only flowing fabricated magnetic structures and controlling magnetic field direction, numerous particles can selectively choose the desired path within microfluidic channels.

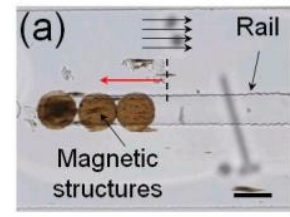

(d) All left direction

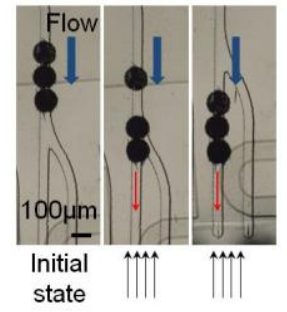

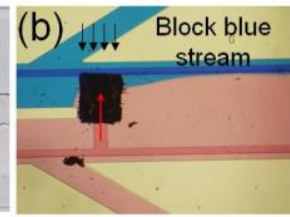

(e) All right direction

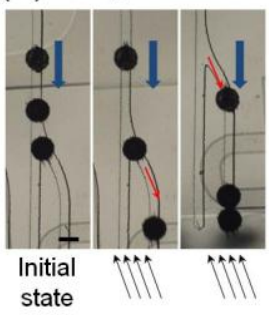

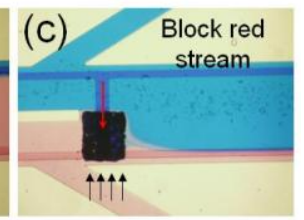

(f) Sorting: selective

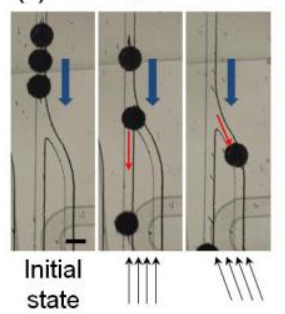

Figure 6. Rail-guided actuations of in-situ fabricated magnetic structures 
By alternatively flowing $\mathrm{MN}$ containing resin and non-magnetic resin into the microfluidic channels, and then fabricating the microstructures, heterogeneous assembly of magnetic and non-magnetic structures is illustrated (Fig. 7(a)). Furthermore, selective magnetic actuation of heterogeneously assembled structures in multiple rails is shown in Fig. 7 (b).

The railed microfluidic process is an innovative technique to guide the movement of in situ photopolymerized microstructures and self-assemble the structures. All guided microstructures are finally blocked and unable to move forward owing to the rail's structural geometry at the end of the rail as in Fig. 7(a). Therefore, the end of the rail works as a barrier to block the movement of the microstructures and to initiate the assembly process.

Heterogeneous assembly is achieved on the basis of multiple rails with the ends. Introduced UV curable resin is alternatively exchanged between $\mathrm{MN}$ containing polymers and non-magnetic resin to allow heterogeneous microstructures fabrication. Thus, magnetic structures and non-magnetic structures are assembled simultaneously at the end of the rails. After the assembly, only magnetic parts are selectively moved due to the applied magnetic fields as demonstrated in Fig. 7(b). A skeleton assembly is composed of non-magnetic parts and magnetic parts. Arms only react to the magnetic force without any control of the flow field after the assembly.

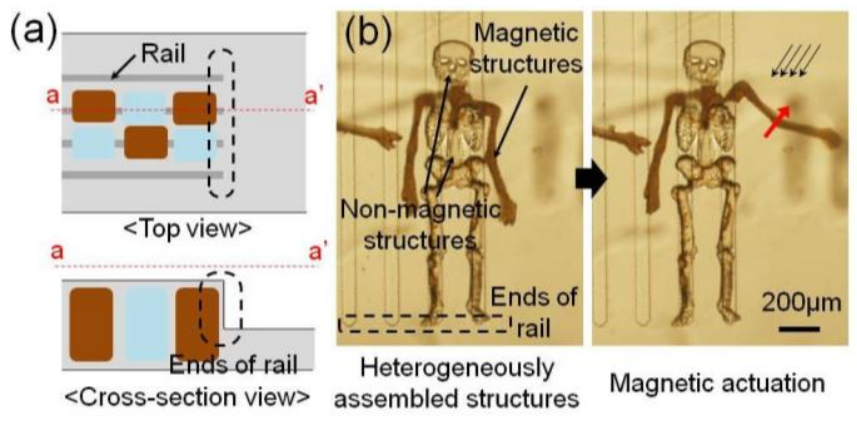

Figure 7. Heterogeneous assembly of magnetic structures and non-magnetic structures, and selective actuation

\section{CONCLUSION}

In this article, we demonstrate in situ fabrication of magnetic actuators via one-mask one-exposure method using optofluidic maskless lithography (OFML) system and their real-time actuation technique. Various magnetic actuators are fabricated using magnetic nanoparticles containing photocurable resin. Both anchored actuator such as microcantilever and unanchored actuator are easily generated with one-step fabrication process. Different from conventional MEMS technology to fabricate anchored microcantilever beam requiring complex steps including sacrificial layer, our technique using UV patterning method only requires one-mask one-exposure which extremely reduces fabrication process time. Both photopatterning and actuation of magnetic structures take place in the same spot using our system. With this system, transportation of fabricated actuator from the fabrication site to actuation site is not necessary. All the actuation is executed by controlling external permanent magnet from the outside of the microfluidic channel. Many types of actuation such as free-floating, bounded, railed actuation and selective actuation are demonstrated within microfluidic channels. Owing to its simplicity and flexibility, we believe one-mask one-exposure magnetic actuator fabrication and actuation method will not only impact current MEMS technology but also encourage innovation in a wide range of application areas in the future.

\section{REFERENCES}

[1] H. Rothuizen, et al., "Fabrication of a micromachined magnetic $\mathrm{X} / \mathrm{Y} / \mathrm{Z}$ scanner for parallel scanning probe applications," Microelectronic Engineering, 53, 509-512 (2000)

[2] H. Guckel, et al., "Fabrication and testing of the planar magnetic micromotor," Journal of Micromechanical Microengineering, 1, 135 (1991)

[3] J. W. Judy, "Microelectromechanical systems(MEMS): fabrication, design and applications," Smart Materials and Structures, 10, 1115 (2001)

[4] J. W. Judy and R. S. Muller, "Magnetically actuated, addressable microstructures," Journal of Microelectromechanical Systems, 6, 249, (1997)

[5] C. Liu and Y. W. Yi, "Micromachined magnetic actuators using electroplated permalloy," IEEE Transactions on Magnetics, 35, 1976 (1999)

[6] J. W. Judy, et al., "Magnetic mciroactuation of polysilicon flexure structures," Journal of Microelectromechanical Systems, 4, 162 (1995)

[7] D. C. Pregibon, et al., "Magnetically and biologically active bead-patterned hydrogels," Langmuir, 22, 5122 (2006)

[8] D. K. Hwang, et al., "Microfluidic-based synthesis of non-spherical magnetic hydrogel microparticles," Lab on a Chip, $8,1640(2008)$

[9] S. E. Chung, et al., "Optofluidic maskless lithography system for real-time synthesis of photopolymerized microstructures in microfluidic channels," Applied Physics Letters, 91, 041106 (2007)

[10] D. Dendukuri, et al., "Modeling of oxygen-inhibited free radical photopolymerization in a PDMS microfluidic device," Macromolecules, 41, 8547 (2009)

[11] S. E. Chung, et al., "Guided and fluidic self-assembly of microstructures using railed microfluidic channels," Nature Materials, 7, 581 (2008)

\section{ACKNOWLEDGEMENTS}

This work was partly supported by the Korea Research Foundation Grant and the Korea Science and Engineering Foundation (KOSEF) grant funded by the Korean Government (MEST) (KRF-2009-220-C00010 and No. 0414-20090036).

\section{CONTACT}

*S. Kwon, tel: +82-2-880-1736; $\underline{\text { skwon@ @nu.ac.kr }}$ 